\title{
Descripción de los Procesos de Evaluación en la Escuela de Medicina de la Pontificia Universidad Católica de Chile: Experiencia de 10 años
}

IGNACIO SÁNCHEZ D. ${ }^{1}$, ARNOLDO RIQUELME P. ${ }^{2}$, RODRIGO MORENO B. ${ }^{3}$, SOFÍA SALAS I. ${ }^{4}$, JULIO PERTUZÉ R. ${ }^{5}$, PATRICIA GARCÍA C. ${ }^{6}$

\section{Description of Assessment Processes at the Medical School of the Pontificia Universidad Católica de Chile: A Ten-year experience}

Globalization of health care seems to be irreversible and beyond cultural differences and local realities; consequently, medical education needs to have a common set of core principles or standards that may be applied worldwide. The aim of participating in assessment processes is to guarantee that medical education takes place in a sufficiently rich environment to promote extensive academic purposes. The Medical School of the Pontificia Universidad Católica de Chile (PUC) participated in three assessment processes that included three stages: internal assessment, external assessment, and accreditation judgment. Two of these assessments were voluntarily carried out following the standards set by the Liaison Committee on Medical Education-LCME, and they took place in 1997 and 2007. The other assessment was based on standards set by the Chilean accrediting organism, the National Committee for Undergraduate Program Accreditation (Comité Nacional de Acreditación de Pregrado-CNAP) and took place in the year 2001. In all three experiences, internal assessment was the most enriching stage, stimulating reflections among students and teachers in order to recognize areas of strengths and weaknesses. External assessment processes, especially those based on international standards, are very important for the institutional and program development of Medical Schools. The PUC Medical School on its whole learnt how to carry out an assessment process and was able to improve several weaknesses without pressure, moving from quality assurance to quality enhancement. The present paper analyzes the major challenges involved in an external assessment process.

(Rev Med Chile 2010; 138: 1055-1061).

Key words: Accreditation; curriculum/standards; medical education; quality assurance.

\author{
'Departamento de Pediatría y \\ ex Decano de la Facultad de \\ Medicina. \\ ${ }^{2}$ Departamento de \\ Gastroenterología y miembro \\ del Centro de Educación \\ Médica de la Escuela de \\ Medicina. \\ ${ }^{3}$ Departamento de \\ Enfermedades Respiratorias \\ y Director del Centro de \\ Educación Médica de la \\ Escuela de Medicina. \\ ${ }^{4}$ Departamento de Obstetricia \\ y Ginecología y Directora del \\ Comité de Ética. \\ ${ }^{5}$ Departamento de \\ Enfermedades Respiratorias \\ y Director de Pregrado de \\ la Escuela de Medicina; \\ 'Laboratorios Clínicos y \\ ex Directora de la Escuela \\ de Medicina, Pontificia \\ Universidad Católica de Chile. \\ Santiago de Chile. \\ Recibido el 2 de noviembre de \\ 2009, aceptado el 27 de julio \\ de 2010. \\ Correspondencia a: \\ Dr. Ignacio Sánchez D. \\ Rector Pontificia Universidad \\ Católica de Chile. \\ Av. Lib. Bernardo O'Higgins \\ 340. Santiago, Chile. \\ Teléfono: (56-2) 354-2346 \\ Fax: (56-2) 354-2211 \\ E-mail: igsan@med.puc.cl
}

E n el mundo moderno, la globalización de la salud pareciera ser irreversible. La revolución de la información, facilitada por Internet, ha permitido que muchas personas manejen conceptos básicos de medicina y por tanto, esperan que sus médicos tengan un nivel de logro profesional similar al de los mejores centros internacionales. Entonces, parece razonable pensar que no obstante las diferencias culturales y las realidades de cada localidad, la educación médica 
requiere de un conjunto de principios básicos o estándares de calidad que puedan ser aplicados mundialmente ${ }^{1,2}$. Junto a lo anterior, la ciencia médica se va unificando con la medicina basada en la evidencia y existe una gran necesidad de garantizar calidad a los usuarios de los sistemas de salud. Por este motivo, en 1999 la World Federation of Medical Educators (WFME) se reunió en Copenhague para definir los Estándares Internacionales en educación médica básica ${ }^{2,3}$. De manera similar, el Liaison Committee on Medical Education (LCME), autoridad acreditadora de los programas de educación médica en Norteamérica ${ }^{4}$, estableció varios estándares que se utilizan para acreditar escuelas de medicina en los Estados Unidos y Canadá. Según esta organización, "la acreditación es un proceso de aseguramiento de la calidad en la educación superior que determina si una institución o programa cumple con los estándares de calidad establecidos para el funcionamiento, estructura $y$ desempeño. El proceso también promueve el mejoramiento institucional y curricular.".

La educación superior en Chile ha experimentado un crecimiento significativo en los últimos veinte años, aumentando tanto el número de universidades privadas como el número de alumnos de pregrado ${ }^{6}$. El número de escuelas de medicina aumentó de 9, en 1986, a 27 en 2009, y el número de alumnos matriculados se duplicó en el mismo período. Este crecimiento explosivo, sin un adecuado programa de aseguramiento de la calidad, hizo necesaria la creación de un proceso de acreditación institucional. Sin embargo, a la fecha, el control de la calidad en Latinoamérica aún se considera insatisfactorio ${ }^{7}$. Sólo una minoría de los países Latinoamericanos posee sistemas de aseguramiento de la calidad basados en evaluación externa, y muchos de ellos utilizan únicamente criterios nacionales generales en la educación superior $^{5}$. Por otro lado, existen instituciones como la Pan American Federation of Associations of Medical Schools (PAFAMS), una organización académica no-gubernamental, que fomenta el intercambio de ideas y experiencias entre sus miembros, asociaciones y escuelas de medicina afiliadas, enfocados en la calidad y profesionalismo de los egresados de las escuelas de medicina latinoamericanas ${ }^{5}$.

La acreditación institucional garantiza que la educación médica se realice en un ambiente adecuado, que promueva el desarrollo de una amplia actividad académica. La acreditación puede obte- nerse sometiéndose a un proceso conformado por tres etapas: auto-evaluación interna, evaluación externa y pronunciamiento de acreditación. En algunos países, como Chile, se trata de un proceso de carácter obligatorio al cual deben someterse todas las escuelas de medicina. En Estados Unidos, a través de una evaluación sistemática, el proceso busca promover y garantizar públicamente la calidad de las instituciones de educación superior y la de sus programas de pre y postgrado ${ }^{4}$. Si bien el LCME sólo acredita los programas de medicina estadounidenses y canadienses, las autoridades de la Escuela de Medicina de la Pontificia Universidad Católica de Chile (EMUC) le solicitaron la realización del proceso de acreditación bajo los mismos estándares de calidad usados en Norteamérica.

Nuestra escuela de medicina fue evaluada por primera vez el año 1997, constituyéndose en la primera escuela de medicina chilena en someterse a una evaluación de aseguramiento de la calidad de nivel internacional. Cuatro años después, la Asociación de Facultades de Medicina de Chile (ASOFAMECh) promovió un proceso de acreditación para la educación médica de pregrado y, entre los años 2000 y 2002, el Comité Nacional de Acreditación de Pregrado (CNAP) evaluó a las escuelas de medicina afiliadas a ASOFAMECh. Posteriormente, en el año 2007, nuestra escuela de medicina se sometió nuevamente a una evaluación, bajo los estándares de calidad definidos por el LCME.

El objetivo de este artículo, es describir los procesos a los cuales se sometió la EMUC, y analizar las ventajas de usar estándares internacionales y nacionales. Se destaca la importancia de la etapa de auto-evaluación, en que se reconocen las fortalezas y debilidades de nuestra escuela de medicina, y que entrega además la oportunidad de corregir estas últimas, pudiendo no sólo garantizar la calidad sino incluso mejorarla.

\section{Descripción de los Procesos de Evaluación}

\section{Proceso de evaluación internacional de 1997}

En la etapa de autoevaluación del primer proceso de evaluación en que se aplicaron los estándares internacionales del LCME, participaron principalmente el Decano en funciones en la época y un grupo de autoridades, médicos e investigadores de la EMUC; la participación de docentes y alum- 
nos fue limitada. El marco de referencia utilizado para evaluar la estructura, función y desempeño del programa curricular de pregrado fueron los estándares aplicados en las escuelas de medicina de los Estados Unidos de Norteamérica ${ }^{8}$. El proceso evaluativo estuvo centrado principalmente en la estructura y funcionamiento del programa curricular de pregrado. Visitó nuestra escuela un equipo integrado por 2 examinadores externos, no hispanoparlantes, con experiencia en acreditación de escuelas de medicina en Norteamérica, quienes se reunieron con las principales autoridades de la EMUC y también con los alumnos, además de visitar las instalaciones educacionales y los campos clínicos. Se contó con el apoyo de una traductora profesional.

\section{Principales hallazgos y resultados de la visita}

Los principales hallazgos, entre los que se cuentan fortalezas y oportunidades de mejoramiento, se muestran en la Tabla 1. Finalizada la visita, nuestra escuela implementó importantes modificacio- nes entre las que cabe mencionar, por ejemplo: a) la creación del Comité de Currículo, integrado por un representante estudiantil y docentes de los cursos básicos, preclínicos y clínicos; b) el mejoramiento significativo en las instalaciones académicas, incluida la construcción del edificio de la Escuela de Medicina y la Biblioteca Biomédica; c) el incremento de la docencia ambulatoria y de la experiencia clínica en psiquiatría, la cual se vio facilitada por la habilitación de un servicio hospitalario psiquiátrico; y d) la consolidación de un Centro de Educación Médica.

\section{Proceso de evaluación nacional de 2001}

El año 2001, la EMUC se sometió al proceso de acreditación nacional de la CNAP. La escuela de medicina presentó su auto-evaluación conforme a las instrucciones señaladas por el comité de la CNAP, bajo la dirección de un miembro de la Facultad especialmente designado para ello. Luego, un equipo de pares evaluadores nombrados por la CNAP visitó la Escuela y, basándose en los criterios

\section{Tabla 1. Fortalezas y oportunidades de mejoramiento del proceso de evaluación internacional 1997}

\section{Fortalezas}

El Decano y su equipo comparten los ideales y filosofía respecto de la educación médica

Existe un fuerte compromiso por reformar el programa curricular de pregrado

Los académicos y estudiantes están convencidos de que su Escuela de Medicina es de excelencia

Las autoridades administrativas y docentes han iniciado una reforma curricular significativa

La Escuela de Medicina está bien administrada desde el punto de vista financiero y brinda un amplio rango de instalaciones propias y asociadas para la docencia

El Hospital universitario es una instalación moderna de la más alta calidad, donde se provee de cuidados a los pacientes y se hace docencia e investigación de primer nivel

\section{Oportunidades de mejoramiento}

Se requiere realizar mejoras en las instalaciones, de modo que permitan el cumplimiento de las reformas curriculares Los espacios destinados al estudio, las salas de clases, laboratorios y biblioteca son insuficientes

Es necesario centralizar el manejo curricular, mediante un Comité de Currículo y ampliar la participación de los académicos y estudiantes en la planificación, implementación y evaluación del programa educativo

Las ciencias del comportamiento, el ciclo de la vida y el desarrollo del niño son aspectos que no están adecuadamente representados en el currículo. La experiencia clínica en psiquiatría es insuficiente

Es necesario ofrecer a los alumnos más oportunidades de estar en contacto precoz con pacientes para reforzar las exposiciones didácticas sobre salud, las ciencias del comportamiento y la relación médico-paciente

La Escuela debe ampliar los criterios de admisión, donde se consideren los atributos no-cognoscitivos y logros personales de los postulantes

Se hace necesario contar con un sistema efectivo de consejería estudiantil 
de evaluación definidos por este organismo, emitió un informe escrito indicando las principales fortalezas y oportunidades de mejoramiento. Algunas de las principales fortalezas descritas por la Comisión dan cuenta de la formación de alta excelencia académica que otorga la carrera de medicina de la EMUC; también destaca a los académicos como idóneos y debidamente calificados para la docencia y el desarrollo de actividades de investigación a nivel de excelencia, y a los estudiantes, quienes son seleccionados con criterios de alta exigencia y que demuestran una actitud positiva frente al aprendizaje. Otra fortaleza es el programa académico, resultado del esfuerzo sostenido de los académicos y directivos de la carrera. También menciona la necesidad de mejorar la efectividad del Comité de Currículo, en especial en lo que se refiere a la implementación de los programas y la coordinación entre las áreas básica, preclínica y clínica. Señala la necesidad de desarrollar métodos docentes más participativos y de homogeneizar la capacitación docente a los monitores, así como hacer más efectiva la capacitación docente en técnicas pedagógicas, incluida la evaluación de competencias clínicas y la docencia práctica. Finalmente, tras analizar los antecedentes reunidos durante el proceso de evaluación, la CNAP acordó acreditar la carrera por un período de 7 años, el máximo período de acreditación en Chile.

\section{Proceso de evaluación internacional de 2007}

En el segundo proceso de evaluación internacional, se estructuró un "Equipo de Evaluación", que trabajó en forma conjunta con el Director de la Escuela y el Director de Pregrado de la época, quienes completaron las bases de datos en áreas como contexto institucional, programa curricular de medicina, estudiantes, académicos, recursos educativos y financieros, conforme a los estándares del LCME. Estas bases fueron luego analizadas por cinco comités que trabajaron en el proceso de autoevaluación, donde participaron los directores de pre y postgrado, los miembros del Comité de Currículo, Profesores Jefes y personal administrativo, llegando a un total de más de 100 académicos y a un número similar de alumnos de pregrado, representando a $15 \%$ del universo. Una vez que cada grupo de estudio hubo finalizado la autoevaluación, se envió a cada uno de los Departamentos de la Escuela, para su análisis, un documento consolidado que destacaba las fortalezas, debilidades y oportunidades de mejoramiento. Las sugerencias y comentarios recibidos se incluyeron en el documento final. En forma paralela, el grupo de estudiantes completó el proceso de autoevaluación desde su propia perspectiva y su documento final fue analizado por los representantes estudiantiles ${ }^{4}$. Un equipo de tres expertos estadounidenses en educación médica (uno de ellos hispanoparlante) visitó durante cuatro días nuestra Escuela, ocasión en que evaluaron el diseño del programa de medicina, su implementación, gestión y metodología de evaluación, conforme a los criterios del LCME. Se contó con el apoyo de una traductora profesional.

\section{Principales hallazgos de la visita}

La Tabla 2 resume sus hallazgos, que incluyen un detalle de las fortalezas institucionales y se mencionan áreas en las que la Escuela cumple en forma parcial o no cumple los estándares del LCME. Entre las principales fortalezas, el equipo de pares evaluadores mencionó a los alumnos, de quienes señalan que son de excelencia académica, comprometidos, dedicados y muy implicados con su quehacer educativo; los académicos, a quienes se les percibe centrados en la educación y el alumno, generosos en su contribución a la educación médica y muy orgullosos de su institución; los recursos clínicos, los pacientes y los campos clínicos fueron considerados muy apropiados para las necesidades curriculares y estudiantiles. La responsabilidad social también fue considerada una fortaleza institucional, la participación por parte de los estudiantes en iniciativas de "aprendizaje y servicio" con un fuerte apoyo institucional fue definido como admirable y ejemplar; la educación médica, a través del Centro de Educación Médica, fue catalogada como un recurso clave en la medida que la Facultad realiza reformas curriculares; el área de investigación fue considerada fuerte y sólida, con una amplia oferta de oportunidades para los estudiantes de participar en la actividad académica. En la Tabla 2 se describen las oportunidades de mejoramiento en las que se destaca, dentro del análisis curricular, que si bien los objetivos propuestos para el currículo fueron considerados adecuados, en ocasiones éstos no estuvieron claros en la estructura y contenidos, y el continuo humanista se presentó poco articulado y no asociado a la práctica clínica. 
Tabla 2. Fortalezas y oportunidades de mejoramiento del proceso de evaluación internacional 2007

\section{Fortalezas}

Los alumnos, que son académicamente hábiles, comprometidos, dedicados y muy implicados con su quehacer educativo Los académicos, a quienes se les percibe centrados en la educación y el alumno, generosos en su contribución a la educación médica, y muy orgullosos de su institución

Los recursos clínicos: pacientes y los campos clínicos son muy apropiados para las necesidades curriculares y estudiantiles Responsabilidad Social: El compromiso por parte de los estudiantes con el "aprendizaje- servicio", apoyado fuertemente por la institución es admirable y ejemplar

La educación médica, a través del Centro de Educación Médica, es un recurso clave en la medida que la Facultad realiza reformas curriculares

El área de investigación es fuerte y sólida, con una amplia oferta de oportunidades para los estudiantes de participar en la actividad académica

\section{Oportunidades de Mejoramiento}

Es necesario contar con una autoridad institucional efectiva que supervise el currículo en su totalidad. Aún existe una cultura departamental, en oposición a un control académico amplio del currículo

La falta de un internado en psiquiatría es una deficiencia significativa

Se carece de una evaluación formativa consistente en un número importante de cursos

La carga horaria de clases en los tres primeros años del programa no da la posibilidad de un estudio autodirigido

Los residentes que supervisan a los alumnos de medicina no están familiarizados con los objetivos educacionales del programa de pregrado

Es necesario abordar la presencia de deshonestidad académica y copia. Además, algunos estudiantes refieren una percepción de maltrato estudiantil, pero no lo informan por temor a represalias

No se evalúan las características no-académicas de los postulantes a la carrera de medicina

Al poco tiempo de haber recibido la visita del comité evaluador, la EMUC desarrolló un plan para mejorar aquellos aspectos señalados por el equipo de expertos. A continuación se mencionan algunos de los proyectos contemplados en este plan:

a) El Comité de Currículo tendrá mayor injerencia en el diseño e implementación curricular, evitando de este modo una influencia excesiva por parte de los Departamentos.

b) Se debe aumentar la exposición clínica de los alumnos a pacientes psiquiátricos, ya sea incrementando los contenidos del internado de Medicina Familiar o - eventualmente- mediante la creación de un internado de Psiquiatría.

c) Existe la necesidad de mejorar el proceso de las evaluaciones formativas.

d) Los residentes y becados conocerán los objetivos que deben aprender los alumnos de pregrado, y se les capacitará en técnicas docentes.

e) Durante la autoevaluación se detectó la per- cepción de un problema de copia por parte de los estudiantes, el que será enfrentado en conjunto con los alumnos, docentes, residentes y personal administrativo.

f) En relación a la "percepción de maltrato estudiantil", se elaboró un plan de trabajo, en ejecución, que dice relación con evaluar el problema referido al maltrato estudiantil.

g) Hay también una iniciativa enfocada a desarrollar un sistema de consejería personal para los alumnos durante el primer año de la carrera.

h) Se han modificado los espacios de estar y recreación para los alumnos en todos los campos clínicos, de modo de cumplir con los estándares requeridos.

\section{Discusión}

Aún cuando nuestra Escuela de Medicina fue acreditada por el máximo período conforme a es- 
tándares locales, tener la experiencia de someterse, además, a un proceso de evaluación internacional ("con criterios internacionales") presenta muchas ventajas. Si bien la preparación del informe de autoevaluación implica mucha dedicación, ambas visitas fueron catalizadoras de cambios en nuestro programa educativo.

A diferencia de otros procesos de acreditación internacional de escuelas de medicina, que están mayormente dirigidos a garantizar que el médico pueda ejercer su profesión en cualquier parte del mundo9 ${ }^{9}$, en nuestro caso lo importante era mejorar la calidad de nuestro programa de educación médica. Por ello utilizamos este proceso para generar un progreso institucional. Desde esta perspectiva, la etapa más importante fue la auto-evaluación -bajo estándares del LCME- de nuestros recursos y del contenido curricular. Cabe señalar que estos estándares se encuentran disponibles en el sitio web del LCME, por lo que cualquier escuela puede realizar una autoevaluación destinando esos recursos académicos. Además, como menciona Hays ${ }^{9}$, la observación y evaluación de estos aspectos por un equipo externo de educadores experimentados, es una oportunidad para contrastar la percepción interna obtenida a partir del proceso de auto-evaluación, con los expertos externos que no tienen una visión sesgada. En nuestra experiencia, pudimos detectar gran parte de las fortalezas y oportunidades de mejoramiento que más tarde fueron confirmadas por el equipo de evaluadores externos. De hecho, dada la amplia difusión y participación que tuvimos en el proceso, los cambios se empezaron a implementar incluso antes de que los pares evaluadores nos visitaran.

En nuestra opinión, el impacto y la relevancia de este último proceso serán similares al generado por el proceso diez años atrás. Respecto del modo en que hemos abordado el aseguramiento de la calidad de nuestra Escuela de Medicina, en los últimos diez años tenemos una experiencia positiva, ya que la CNAP otorgó a nuestra Escuela el máximo período de acreditación. Adicionalmente, desde el año 2003 los egresados de la EMUC han obtenido los más altos puntajes en el Examen Médico Nacional, pudiendo esto ser un resultado de los cambios curriculares iniciados como parte del proceso de aseguramiento de la calidad previamente descrito ${ }^{10}$.

La principal fortaleza de un proceso de aseguramiento de calidad es la etapa de autoevaluación, que en sí misma estimula la reflexión entre los alumnos y docentes en torno al reconocimiento de fortalezas y debilidades. El proceso vivido el año 1997, nos ayudó a conocer nuestro nivel respecto de los estándares internacionales. Si bien esta evaluación no tuvo carácter obligatorio, aprendimos a llevar adelante un proceso de acreditación que nos permitió detectar nuestras debilidades, pudiendo no sólo garantizar la calidad sino incluso mejorarla. Para dar respuesta a las "Oportunidades de Mejoramiento" indicadas en el informe entregado por el LCME el año 1997, la EMUC participó en concursos gubernamentales nacionales que financian proyectos referidos al mejoramiento de la educación superior y obtuvo el aporte financiero para la construcción de un nuevo edificio para la Escuela de Medicina, una nueva biblioteca biomédica y la creación de un Centro de Educación Médica. Los resultados de la evaluación externa fueron un apoyo importante en la obtención de estos fondos ${ }^{11}$. Los resultados obtenidos por la EMUC en el proceso de acreditación nacional pudieran estar asociados a los cambios motivados por el informe del LCME y a la experiencia ganada por los miembros de la Facultad en la evaluación anterior por parte del LCME. En los años siguientes, el Comité de Currículo, con el apoyo del Centro de Educación Médica, continuó mejorando la calidad de la docencia; por ejemplo, se incorporaron en forma progresiva más sesiones de docencia ambulatoria y se modernizó la metodología de evaluación.

La World Federation for Medical Education (WFME) puso en el tapete el tema del reconocimiento internacional de instituciones y programas de educación médica y realizó una revisión sistemática del aseguramiento de la calidad internacional de la educación médica, que incluye procedimientos de acreditación y metodología de aseguramiento de calidad alternativa (Tabla 3 ). La WFME promueve la creación de procedimientos de acreditación nacional; sin embargo, cuando no es factible llevar a cabo un proceso de acreditación, se recomienda trabajar con pares en programas de capacitación ${ }^{12}$. Este escenario es comparable con el contexto chileno en la primera evaluación internacional a la que se sometió la EMUC en 1997. Incluso habiendo iniciado un proceso de acreditación nacional el año 2001, consideramos igualmente útil la evaluación de expertos extranjeros como parte de nuestro sistema de aseguramiento de la 


\section{Tabla 3. Ideas principales en un proceso de Evaluación Internacional}

- La globalización de la salud parece ser irreversible y trasciende a las diferencias culturales y realidades de cada localidad

- La educación médica requiere de un conjunto de principios básicos o estándares que puedan ser aplicados mundialmente

- La auto-evaluación fue la etapa más enriquecedora, ya que estimuló la reflexión de alumnos y docentes en torno al reconocimiento de fortalezas y debilidades

- Los procesos de evaluación externa, particularmente aquellos que se basan en estándares internacionales, son muy importantes para el desarrollo institucional y curricular de las escuelas de medicina

- La continua evaluación del currículo permite a las Escuelas de Medicina abordar sus debilidades sin presión, permitiéndoles garantizar la calidad e incluso mejorarla

Adaptado de WMFE. Task force on defining standards in basic medical education. Report of the working party. Med Educ 2000; 34: 665-75.

calidad, porque ofrece una perspectiva externa con estándares internacionales.

En el futuro y de acuerdo a Hodges y Segouin ${ }^{13}$, el desafío para los educadores e instituciones médicas es establecer un conjunto de estándares internacionales que acoja las diferencias que existen en este ámbito entre continentes, países y culturas. Hacia este objetivo se deben orientar los procesos de certificación y acreditación en Educación Médica.

\section{Referencias}

1. Cohen JJ. Defining international standards in basic medical education: the World Federation for Medical Education has initiated a timely discussion. Med Educ 2000; 34: 600-1.

2. WFME. Task force on defining international standards in basic medical education. Report of the working party. Med Educ 2000; 34: 665-75.

3. Karle H. Global standards and accreditation in medical education: a view from the WFME. Acad Med 2006; 81: S43-8.

4. LCME. Liaison Committee on Medical Education Accreditation Standards 2008. Disponible en www.lcme.org/ standard.htm

5. Pulido MP, Cravioto A, Pereda A, Rondon R, Pereira G.
Changes, trends and challenges of medical education in Latin America. Med Teach 2006; 28: 24-9.

6. Sánchez I, Riquelme A, Moreno R, Mena B, Dagnino J, Grebe G. Revitalising medical education: the School of Medicine at the Pontificia Universidad Católica de Chile. The Clinical Teacher 2008; 5: 1-5.

7. Goic A. Proliferación de escuelas de medicina en Latinoamérica: causas y consecuencias. Rev Med Chile 2002; 130: 917-24.

8. Kassebaum DG. LCME accreditation standards for management of the medical school curriculum: a clarification. Liaison Committee on Medical Education. Acad Med 1994; 69: 37-8.

9. Hays R. International accreditation of medical schools. Med Educ 2003; 37: 662.

10. Bitran $M$, Wright $A$, Zúñiga $D$, Mena B, Velasco N, Moreno R. Mejoría en el desempeño académico de estudiantes de medicina en tiempos de reforma curricular. Rev Med Chile 2002; 130: 437-45.

11. Enríquez LO, Mena CB. Habilitación profesional. Condiciones para el aseguramiento de la calidad de la educación médica y condiciones para la confianza recíproca: Experiencia y visión de ASOFAMECH. Rev Med Chile 2005; 133: 483-94.

12. Karle H. International recognition of basic medical education programmes. Med Educ 2008; 42: 12-7.

13. Hodges BD, Segouin C. Medical education: it's time for a transatlantic dialogue. Med Educ 2008; 42: 2-3. 